\section{Asthmareaktionen nach Weingenuss - Ätiologie weiter unklar}

\author{
Etwa ein Drittel aller Asthmapatienten berichtet über eine Zunahme \\ der Symptome nach Weingenuss. Bisher schlugen Versuche fehl, die \\ Ätiopathogenese dieser Reaktionen zu klären. Australische Pneumo- \\ logen unternahmen jetzt einen neuen Versuch mit Fokus auf den \\ Sulfitgehalt des Weines.
}

\begin{abstract}
A cht Patienten mit Asthma bronchiale, die in der Anamnese eine Verschlimmerung der Erkrankung nach Weingenuss angegeben hatten, nahmen an einem Cross-over-Provokationsversuch mit doppelter Verblindung teil. Sie mussten dabei jeweils ein vorgegebenes Volumen an Wein trinken, der entweder niedrige oder hohe Sulfitkkonzentrationen aufwies. Die Probanden tranken die Weine im Abstand von 48 Stunden. Zur Wahl standen Rot- oder Weißweine, dabei wurde der Weintyp gewählt, auf den die Patienten nach eigenen Angaben schlimmer reagierten. Die Weine mit wenig Sulfit stammten aus dem Handel, die Hochdosis-SulfitWeine (250-300 ppm) entstanden durch Zugabe von Natriumdisulfit. Gemessen wurden die Einsekundenkapazität $\left(\mathrm{FEV}_{1}\right)$ sowie die bronchiale Hyperreaktivität (Provokation mit Histamin bis zur 20\%igen Reduktion von $\mathrm{FEV}_{1}$ )
\end{abstract}

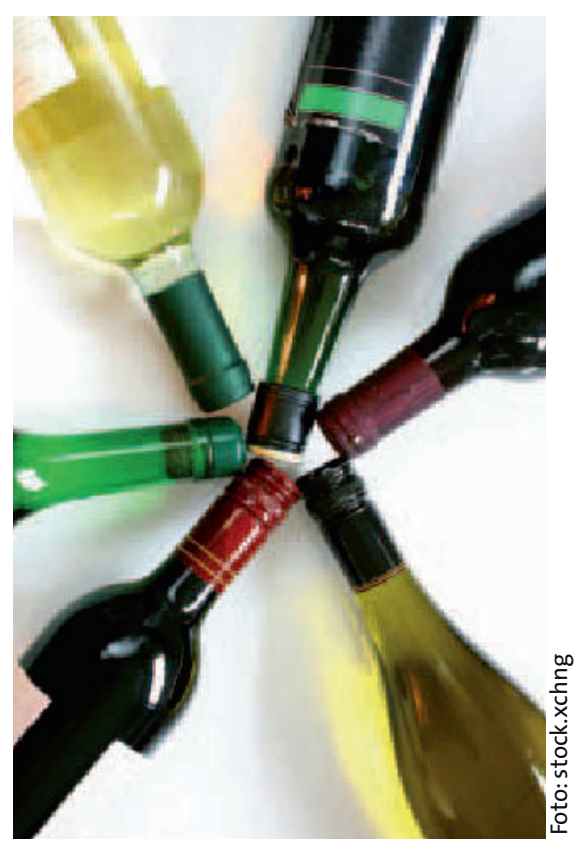

Ob rot, ob weiß - die Rolle von Wein als Asthmaauslöser bleibt unklar.
- jeweils vor und nach dem Trinken des Weins.

Keiner der Studienteilnehmer zeigte einen klinisch signifikanten $\mathrm{FEV}_{1}$-Abfall nach dem Genuss von $150 \mathrm{ml}$ Wein, getrunken innerhalb von fünf Minuten. Dabei war es auch unerheblich, ob es sich um Wein mit hohem oder niedrigem Sulfitgehalt handelte. Veränderungen der bronchialen Hyperreagibilität nach Weingenuss wurden zwar beobachtet, die Änderungen waren aber uneinheitlich und machten sich in einem Fall sogar als Besserung bemerkbar.

Die Autoren folgern aus der Studie, dass das Additivum Sulfit keinen oder nur einen geringen Einfluss auf die respiratorischen Symptome der Patienten nach Weinkonsum hat. Vielmehr dürften Reaktionen auf Wein bei Asthmatikern komplexe Ursachen mit mehreren Cofaktoren haben, die im Einzelfall sehr unterschiedlich sind.

Fazit: Weingenuss hatte bei Asthmapatienten keinen Abfall von $\mathrm{FEV}_{1}$ zur Folge, der Einfluss auf die bronchiale Hyperreagibilität fiel in diesem kleinen Studienkollektiv uneinheitlich aus.

$b k$

Vally $\mathrm{H}$ et al. Changes in bronchial hyperresponsiveness following high- and lowsulphite wine challenges in wine-sensitive asthmatic patients. Clin Exp Allergy 2007; 37: 1062-6

Mit durchschnittlich 1,6 Verordnungen pro Rezept erhielten die Patienten insgesamt ca. 2,5 Mio. Autoinjektoren im Jahr 2004. Im Durchschnitt ergab sich damit eine Verordnungszahl von 5,71 Injektoren pro 1.000 Einwohner. Auffällig war ein deutliches Nord-Süd-Gefälle: In den nordöstlichen Staaten lag die Zahl der verordneten Autoinjektoren zwischen 8 und 12 je 1.000 Personen, während im Südwesten nur zwischen 2 und 3 pro 1.000 Einwohnern rezeptiert wurden. Die niedrigste Verordnungsrate mit 2,7/1.000 ergab sich für Hawaii.

Als Erklärung für den beobachteten Unterschied weisen die Autoren auf eine mögliche Assoziation zwischen niedrigen Vitamin-D-Spiegeln und erhöhtem Allergierisiko hin. In den Staaten mit gerin- 